\title{
AIS Data Collection, Storage and Display System
}

\author{
Yi Jiang ${ }^{1,2, a}{ }^{*}$, Da Li ${ }^{2, b}$ and Shufang Zhang ${ }^{2, c}$ \\ ${ }^{1}$ School of Electronic and Information Engineering, Beihang University, Beijing, China \\ ${ }^{2}$ Information Science and Technology College, Dalian Maritime University, Dalian, China \\ a*j_y@dlmu.edu.cn, blddlmu@163.com, csfzhang@dlmu.edu.cn
}

Keywords: Data collection, Data storage, Data display, ARM9, NandFlash, LCD.

\begin{abstract}
In an automatic identification system (AIS) receiver, after frequency down conversion and carrier separation, the frequency of AIS signals becomes $9.6 \mathrm{KHz}$. The design of AIS data collection, storage and display system based on ARM9 is presented for the data storage and playback of waveform in the paper. First, analog AIS signals are converted into digital signals by the analog-digital (AD) module of mini2440. Then, the digital signals are saved into the NandFlash. Next, the data can be displayed on the liquid crystal display (LCD). Furthermore, data from the NandFlash could send to the computer for handling later.
\end{abstract}

\section{Introduction}

The automatic identification system (AIS) is the communication system, which is widely used in the in the maritime domain. In the rapid development of electronic measurement and digital technology, the data collection technology is an important means of obtaining data information. Data collection is an interface which collects data from the outside of the system. It collects non-electric quantity or power signals from the sensors and other equipment automatically for handling later, such as analog and digital measured unit ${ }^{[1]}$. The data storage technology records the data stream in the processing with a specified format in the internal or external storage medium of the computer. Data playback technology reproduces the data by reading the data in a storage medium $^{[2]}$.

The experiments based on AIS in the sea are not convenient. The AIS data collection, storage and display system is developed, whose function includes the data collection, the data storage, the data playback, the serial port communication and the spectrum analysis. It is comprised of the AD, the NandFlash, the serial port and the LCD based on ARM2440. With the advantage of the small size and convenient operation, it is portable used for the collection and processing of AIS analog modulation signal, which frequency is $9.6 \mathrm{KHz}$. Thus, it has great application value.

\section{System Structure}

This paper designed the AIS data collection storage system based on ARM2440 of Samsung. The function includes AIS data collection, storage and playback. Meanwhile, the stored data can be sent to the computer via a serial port for the spectrum analysis or AIS message analysis. ARM is the main controller, which controls the entire system and assigns the clock of crystal oscillator of the AD, the NandFlash, the serial port and the LCD. The transformed data are saved in the NandFlash. Then the AIS data can be read from the NandFlash by the LCD module for the display. Furthermore, the data can also be sent to the computer via the serial port. These data can be used for spectrum analysis or AIS message analysis. The structure of the system is illustrated in Fig. 1. 


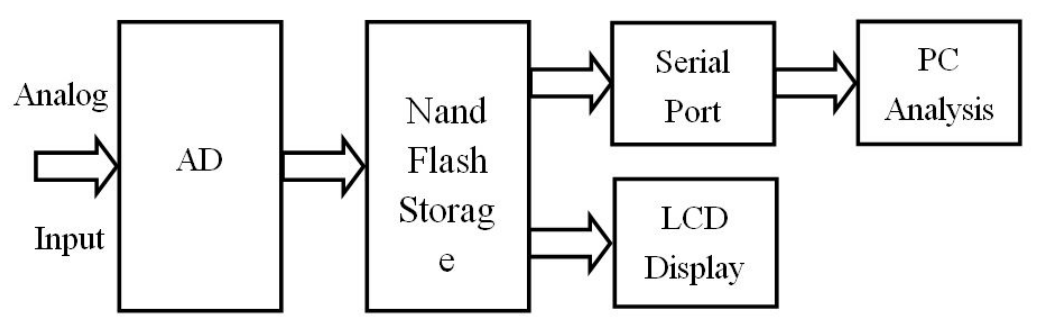

Fig. 1 System Composition Structure

\section{System Function Realization}

The whole system is comprised of the data collection module, the control module, the data storage module, the data transmission module and the data display module. The software design consists of five modules to complete the data collection, storage and display. The AIS data is collected by the data collection module by AD. The control module and the data storage module are used for data storage by the NandFlash. The data transmission module and the data display module are used for the data dynamic display by the LCD and handling later, such as the spectrum analysis, AIS message analysis. The implementation and the design of these modules are explained as following.

Design of Control Module. For the realization of the AIS data collection and storage and display, the ARM needs to coordinate all these five modules. The software design of the control module based on the ARM is shown in Fig. 2. First, the collected data is converted by AD. And high and low 8 bits are written into NandFlash in the proper order. And then determine whether the capacity reaches the value that has been set. If it does, the LED will light and it will stop writing data. If it does not, it will continue to write data. Finally, the AIS data is displayed on the LCD after the storage. Meanwhile, the data can also be transmitted to the computer by the serial port.

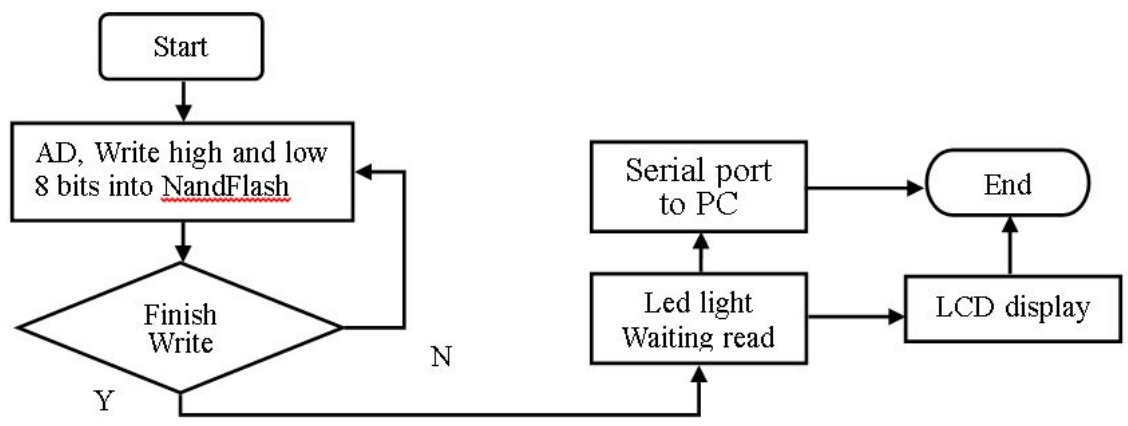

Fig. 2 Software Design of Control Module

Data Collection Module. The collected AIS data of $9.6 \mathrm{KHz}$ is the AIS signal after frequency down conversion and carrier separation. The AD of S3C2440 has a maximum conversion frequency of $500 \mathrm{KHz}$, which meets the requirement of AIS data collection. The AD of S3C2440 can collect 4 channels of external input signals to process. The main features include the resolution of 10, the analog input range of 0 to $3.3 \mathrm{~V}$; maximum switching frequency of $500 \mathrm{KSPS}$.

The data collection module is mainly complete the AIS data collection by the AD. First, for setting the sampling frequency, the PRSCVL of AD control register (ADCCON) is changed to divide the frequency of PCLK. Then, automatically convert read mode is set and the data transformmtion begins. Next, the query model is used to determine whether the transformation is finished ${ }^{[3]}$. Finally, after the AD transform, digital signals with 10 bits are output.

Data Storage Module. The NandFlash is mainly used for large capacity storage, with high cost performance, large capacity and small volume. The NandFlash of K9F1G08 is used in this system. The largest number of write is one million. The bit wide is 8 and the capacity is $256 \mathrm{M}$. The size of page is $(2 \mathrm{~K}+64)$ bytes $^{[4]}$.

The data storage module is designed to read data from the collection module as the storage buffer. Because the NandFlash width is 8 bits and AD digital output is 10 bits, the 10 bits digital data is 
divided into high and low 8 bits and written into the NandFlash. The size of the NandFlash is $256 \mathrm{M}$. It can store data about $256 \mathrm{M}$ at most in theory if the data is written from the first block.

Data Transmission Module. The S3C2440 provide three separate asynchronous serial I/O. Each UART contains a baud rate generator, a sender, a receiver and a control unit. Each UART can work in the interrupt mode or DMA mode. In order to increase the efficiency, the interrupt mode is used for receiving data and the query mode is used for sending data in this design.

Data Display Module. The P35-1312 liquid crystal display with a 240X320 resolution is used. The data display module is mainly responsible for the dynamic display of data stored in the NandFlash. The resolution of the $\mathrm{AD}$ is 10 bits, so the range of the input signal is 0 to 1024. In order to suitably quantify the range of screen display, the data is multiplied by 0.23 (240/1024). In addition, for the convenience of observing the part and the full waveform, data are read from the NandFlash every $\mathrm{N}$ points. The horizontal axis size which represents the range of AIS data is changed with different N.

To avoid the phenomenon of screen flicker, partial refresh is used in the design. The data of the first screen is shown and stored. Then before the display of the second screen, data is compared with the first panel data. Just update the changed area ${ }^{[5]}$.

\section{Verification and Testing}

AIS signals after carrier separation are used as an external data source to verify the function of the design, as shown in Fig. 3.

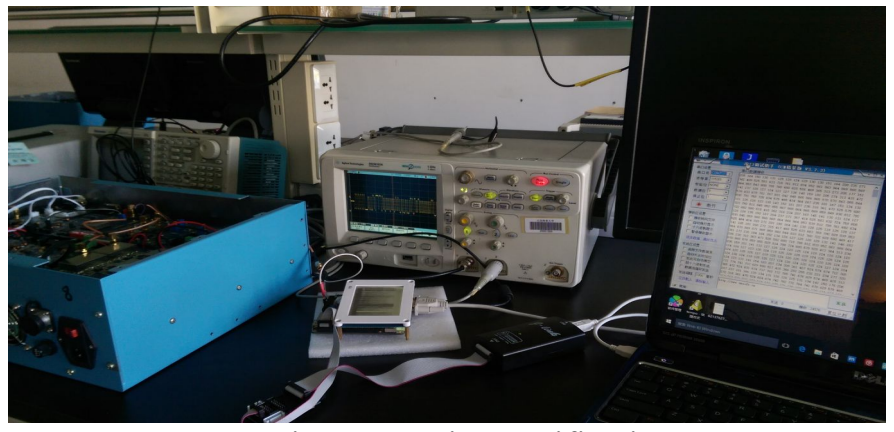

Fig. 3 Function Verification

First, the AIS receiver is started. Then the navigation message data of AIS receiver with the frequency of $9600 \mathrm{~Hz}$ is connected to the data collection module as the input signal for AD conversion. The AIS waveform can be observed by the MATLAB analysis on the computer, the oscilloscope and the display on the LCD.

The converted data is divided into the high and low 8 bits, and then is stored on the NandFlash. The data is read from the NandFlash by the data transmission module firstly. And then send the data to the computer via serial port. Fig. 4 show the data reception by the CommAssistant. We can see the AIS data roughly change as the wave of sine by serial port.

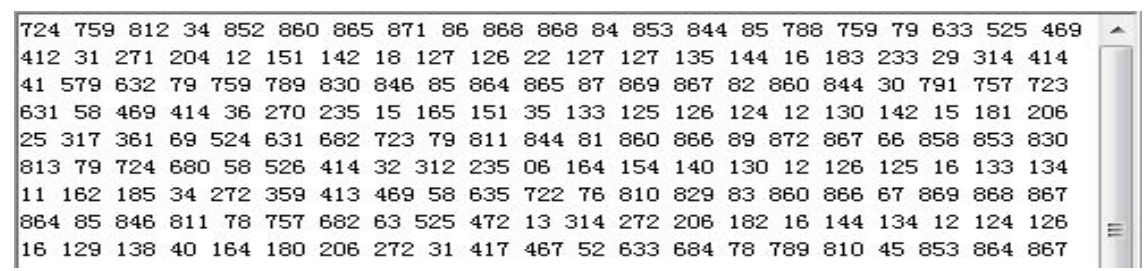

Fig. 4 Reception of Data

For further precise analysis, the signal time domain and spectrum analysis are performed by the MATLAB in the computer. The waveform in the time domain for 600 points and the frequency domain by the FFT is shown in figure 5. We can see that the center frequency is $9601 \mathrm{~Hz}$ which is equal to $9.6 \mathrm{KHz}$ approximately.

The collected AIS signals belong to the data link layer. Non return to zero inverse (NRZI) is used in the communication circuit to fix the code pattern. The training sequence used for the synchronization is composed by 24 bits of alternate 01 . After that, the start flag is consists of 8 bits 
(01111110). The front of NRZI codes is parsed out in Fig. 7, including the training sequence and the start flag. The waveform of AIS can be observed by the LCD when N=2, which is verified by the AIS data given by the oscilloscopes, as shown in Fig. 6.

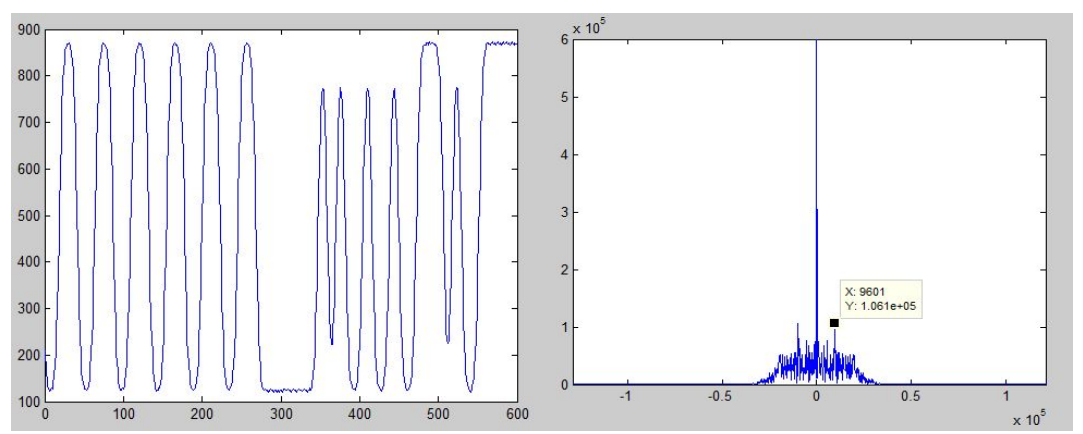

Fig. 5 Waveform of Time Domain (left) and Frequency Domain (right)

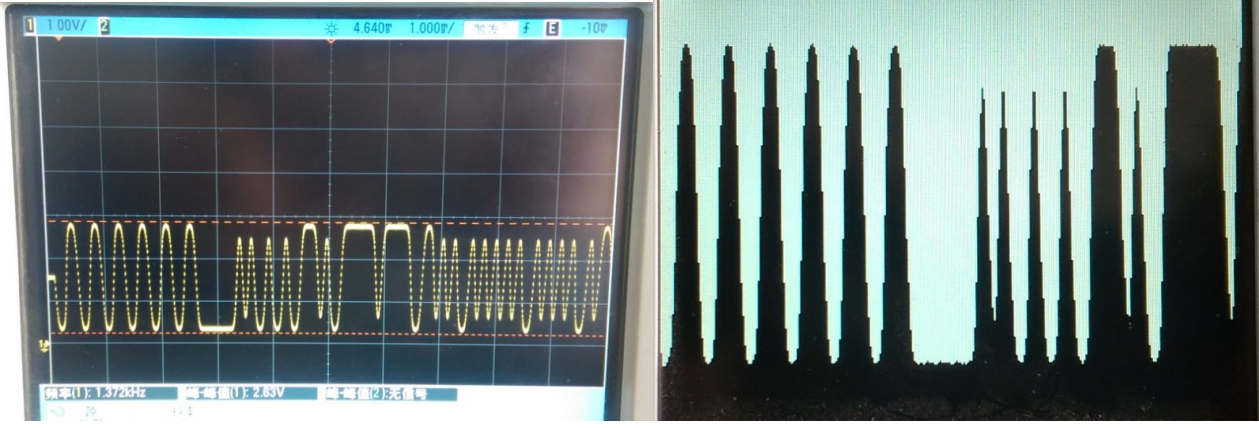

Fig. 6 Waveform Comparison between Oscilloscope (left) and LCD (right)

\section{Conclusion}

This design is composed of the data collection module, the control module, the data storage module, the data transmission module and the data display module by AMR 9 for the data storage and playback of waveform of the AIS data. Its advantage is portability, large storage, plug and play, LCD display etc. Thus, it has great application value in the field of AIS experiments.

\section{Acknowledgments}

This research was partially supported by the Chinese National Science Foundation (No. 61231006 and 61501079), Foundation of Liaoning Educational Committee (No. L2015059), Liaoning Provincial Natural Science Foundation (No. 2014025002) and the Fundamental Research Funds for the Central Universities (No. 3132014329).

\section{References}

[1]Haining Guo. The Explore of Data Acquisition Technology[A]. Mechanical engineering society in yunnan, 2013.

[2]Jiannong Wang, Wei Wang, Zhou Chang. The Common Data Acquisition System Based On Arm9[A]. Proceedings of IEEE 2011 10th International Conference on Electronic Measurement and Instruments (ICEMI'2011) VOL.03, 2011.

[3]Qing Hu, Xiujuan Li, Shufang Zhang. Study of AIS Beacon Control System Based on ARM[A]. International Conference on Environment Engineering and Computer Application, 2014.

[4]Shiyi Wang. The Data Storage System based on ARM[D]. Taiyuan university of science and technology, 2009.

[5] Zhang Ran. The Design of Keyboard and LCD in the Embedded Data Acquisition System[A]. Agricultural engineering society of China, 2005. 